\title{
ON THE MODULE STRUCTURE OF A p-EXTENSION OVER A p-ADIC NUMBER FIELD
}

\author{
YOSHIMASA MIYATA
}

Throughout this paper, let $p$ be an odd prime. Let $k$ be a $\mathfrak{p}$-adic number field and $\mathfrak{o}$ be the ring of all integers in $k$. Let $K / k$ be a finite totally ramified Galois $p$-extension of degree $p^{n}$ with the Galois group $G$. Clearly the ring $\mathfrak{O}$ of all integers in $K$ is an $\mathfrak{o}[G]$-module. In the previous paper [4], we studied $o[G]$-module structure of $\subseteq$ in a cyclic totally ramified $p$-extension, and we have obtained the condition for $\subseteq$ to be an indecomposable $o[G]$-module. In the present paper, we shall prove the following theorem.

THEOREM 1. Suppose that $k$ contains a primitive $p$-th root of unity. Let $K / k$ be a totally ramified Galois p-extension of degree $p^{n}$ such that the extension $K / k$ is not cyclic. Let $E$ be a central idempotent of the group ring $k[G]$ such that $E \subseteq \subseteq Ð$. Then we have $E=1$.

As an immediate consequence of Theorem 1, we have the next theorem.

TheOREM 2. Let $k$ and $K / k$ be as stated in Theorem 1. In addition, we assume that the extension $K / k$ is abelian. Then the $\mathrm{o}[G]$-module $\bigcirc$ is indecomposable.

In $\S 1$, we shall study properties of central idempotent. In $\S 2$, recalling properties of ramification numbers, we shall obtain some inequalities. In $\S 3$, we shall study the special case where the Galois group $G$ is an elementary abelian group of order $p^{2}$. In $\S 4$, we shall study the case where the Galois group $G$ is a direct product of two cyclic groups whose orders are $p$ and $p^{n}$ respectively. In $\S 5$, we shall prove Theorem 1 and Theorem 2.

Received July 25, 1978. 
1.

In this section, we shall study some properties of central idempotents. Let $G$ be a non-cyclic $p$-groups and $H$ be a normal subgroup of order $p$. The natural map from $G$ onto the factor group $G / H$ induces the ring homomorphism $f_{H}$ from the group ring $k[G]$ onto $k[G / H]$. Let $C(G)$ denote the center of $G$. First, we assume that $C(G)$ is not cyclic. Then $C(G)$ contains an elementary abelian $p$-group $C$ of order $p^{2}$.

Lemma 1. Let $G$ be a non-cyclic p-group and $C$ be as stated in the above. Suppose that the center $C(G)$ of $G$ is not cyclic. Let $E$ be a central idempotent of $k[G]$ such that $f_{H}(E)=1$ for any normal subgroup $H$ of order $p$ in $C$. Then $E=1$.

Proof. Without any loss of generality of proof, we can assume that $k$ is the splitting field for $G$. Let $\chi$ be an absolutely irreducible character and $E_{\chi}$ be the central idempotent corresponding to $\chi$. Then there is some subgroup $H$ of $C$ such that $E_{x} \cdot \frac{1}{p}\left(\sum_{h \in H} h\right)=E_{x}$. Since $f_{H}(E)=1$ from the assumption, we have $E=\frac{1}{p}\left(\sum_{h \in H} h\right)+E^{\prime}$, where $E^{\prime}$ is a central idempotent such that $E^{\prime} \cdot \frac{1}{p}\left(\sum_{h \in H} h\right)=0$. Therefore, for any absolutely irreducible character $\chi, E_{\chi} \cdot E=E_{\chi}$, which implies that $E=1$.

Next, we assume that $C(G)$ is cyclic. Clearly $G$ has the unique normal subgroup $Z$ of order $p$, and $G$ is not abelian because $G$ is not cyclic. Then, since $p$ is odd, it is well known that $G$ contains a normal elementary abelian subgroup $B$ of order $p^{2}$ (for example, see [2] III 7.5, p. 303). From the uniqueness of $Z$, it follows easily that $Z$ is contained in $B \cap C(G)$. Let $b$ and $z$ be fixed generators of $B$ such that $b \notin C(G)$ and $z \in C(G)$. Let $C_{G}(b)$ be the centralizer of $b$ in $G$ and $\Phi$ be the Frattini subgroup of $G$. As $B$ is normal in $G$ and $Z$ is a characteristic subgroup of $B$, for any $g \in G$, we have

$$
b^{-1} g b=g z^{i}
$$

for some rational integer $i(0 \leqq i<p)$ which depends on $g$. Since $b \notin C(G)$, from (1), we see easily that $C_{G}(b)$ is a proper normal subgroup of $G$ and hence we have

$$
C_{G}(b) \Phi \neq G
$$


Now we obtain the following lemma.

Lemma 2. Let $G$ be a non-cyclic p-group. Suppose that the center $C(G)$ of $G$ is cyclic. Let $Z, B, z$ and $b$ be as above. Let $E$ be a central idempotent of $k[G]$ such that $E \cdot \frac{1}{p}\left(\sum_{i=0}^{p-1} z^{i}\right)=0$. Then $E$ belongs to the group ring $k\left[C_{G}(b) \Phi\right]$.

Proof. We can also assume that $k$ is a splitting field for $G$ without loss of generality of proof. Let $\chi$ be an absolutely irreducible character and $E_{\chi}$ be the central idempotent of $k[G]$ corresponding to $\chi$ such that $E_{\chi} E=E_{\chi}$. Since $E \cdot \frac{1}{p}\left(\sum_{i=0}^{p-1} z^{i}\right)=0$, we have $E_{\chi} \cdot \frac{1}{p}\left(\sum z^{i}\right)=0$. Let $E_{\chi}$ $=\sum_{g \in G} \alpha_{g} g$, where $\alpha_{g}$ is in $k$. In order to prove the lemma, it is sufficient to show that if $\alpha_{g} \neq 0$, then $g \in C_{G}(b) \Phi$. As is well known, $G$ is an $M$ group and so $\chi$ is induced by a linear character $\alpha$ of some subgroup $A$ in $G$. Denote by $|A|$ the order of $A$. Using $\alpha$, we define a mapping $\dot{\alpha}$ by

$$
\begin{array}{ll}
\dot{\alpha}(g)=\alpha(g) & \text { if } g \in A \\
\dot{\alpha}(g)=0 & \text { if } g \notin A .
\end{array}
$$

Then we have the formula

$$
\chi(g)=\frac{1}{|A|} \sum_{h \in G} \dot{\alpha}\left(h^{-1} g h\right) \quad \text { (for example, see [2] p. 553). }
$$

Now, as $\alpha_{g} \neq 0, \chi\left(g^{-1}\right)=0$ and for some $h_{0} \in G, h_{0}^{-1} g^{-1} h_{0} \in A$. Let $a=h_{0}^{-1} g^{-1} h_{0}$. Immediately, $\chi(a)=\chi\left(g^{-1}\right)$ and so $\chi(a) \neq 0$. Here suppose that $a \notin C_{G}(b)$. Clearly $b \oplus C_{G}(a)$. Since $B$ is normal, it follows easily that $C_{G}(a) B$ is a subgroup of $G$ and $C_{G}(a)$ is a normal subgroup of $C_{G}(a) B$. Hence the set $\left\{1, b, \cdots, b^{p-1}\right\}$ is a set of right coset representatives of $C_{G}(a)$ in $C_{G}(a) B$. Let a set $\left\{h_{1}, \cdots, h_{l}\right\}$ be a set of right representatives of $C_{G}(a) B$ in $G$, so the set $\left\{b^{i} h_{j} \mid 0 \leqq i<p, 1 \leqq j \leqq l\right\}$ are right representatives of $C_{G}(a) B$ in G. As $a \notin C_{G}(b), b^{-1} a b=a z^{i_{0}}$ for some $i_{0}$ such that $i_{0} \neq 0$. For the sake of simplicity, we denote by $z$ the element $z^{i}$ again. Then $b^{-i} a b^{i}=a z^{i}$ for $0 \leqq i<p$. Hence we have

$$
\begin{aligned}
\chi(a) & =\frac{\left|C_{G}(a)\right|}{|A|} \sum_{i, j} \dot{\alpha}\left(h_{j}^{-1} b^{-i} a b^{i} h_{j}\right) \\
& =\frac{\left|C_{G}(a)\right|}{|A|} \sum_{j} \sum_{i} \dot{\alpha}\left(h_{j}^{-1} a h_{j} z^{i}\right) .
\end{aligned}
$$


As $E_{x} \cdot \frac{1}{p}\left(\sum z^{i}\right)=0, z E_{x}=\theta E_{x}$, where $\theta$ is a primitive $p$-th root of unity. Therefore $Z$ is contained in $A$, and so $h_{j}^{-1} a h_{j} z^{i} \in A$ if and only if $h_{j}^{-1} a h_{j}$ $\in A$. Hence we obtain for any $j$

$$
\sum_{i} \dot{\alpha}\left(h_{j}^{-1} b^{-i} a b^{i} h_{j}\right)=\dot{\alpha}\left(h_{j}^{-1} a h_{j}\right)\left(\sum_{i=0}^{p-1} \theta^{i}\right)=0 .
$$

Then, if $a \notin C_{G}(b), \chi\left(g^{-1}\right)=\chi(a)=0$, which is a contradiction. Hence we conclude that if $\alpha_{g} \neq 0$, then $a \in C_{G}(b)$. As $G / \Phi$ is abelian, then $g \in a^{-1} \Phi$. Therefore we have $g \in C_{G}(b) \Phi$, which completes the proof.

2.

Now denote by $e$ the absolute ramification index of $k$. Let $F / k$ be a cyclic ramified extension of degree $p$ with the first ramification number $b$. Define a function $m$ by $m(b)=\left[\frac{(p-1)(b+1)}{p}\right]$. We write $b$ in the form $b=p\left[\frac{b}{p}\right]+p-\lambda$. From [1] Theorem 3, we have that for $(b, p)=1$,

$$
m(b)+\lambda-1 \equiv 0 \quad(p-1) .
$$

Next let $K_{1}$ and $K_{2}$ be cyclic ramified extensions of degree $p$ with ramification numbers $b_{1}$ and $b_{2}$ respectively. Let $K$ be the composition field of $K_{1}$ and $K_{2}$. According to the result of E. Maus ([3]), we can obtain the first ramification number $b\left(K / K_{1}\right)$ for the extension $K / K_{1}$ as follows:

i) if $b_{2}>b_{1}, b\left(K / K_{1}\right)=b_{1}+p\left(b_{2}-b_{1}\right)$

ii) if $b_{2}<b_{1}, b\left(K / K_{1}\right)=b_{2}$

iii) if $b_{2}=b_{1}$, either $b\left(K / K_{1}\right)=b_{1}$, or for some $c$ such that $c<b_{1}$, $b\left(K / K_{1}\right)=c$.

Using these equalities, we shall have the following lemma.

LEMMA 3. Let $K / k$ be a totally ramified extension such that the Galois group of $K / k$ is an elementary abelian p-group of order $p^{2}$. Let $F$ be a subfield of degree $p$ in $K$. Then $m(b(K / F))<p e$.

Proof. There is a subfield $F_{1}$ of degree $p$ such that $K$ is the composition field of $F$ and $F_{1}$. Denote by $b$ and $b_{1}$ the first ramification numbers of $F$ and $F_{1}$ respectively. First, we consider the case $b<b_{1}$. From the above equality i), we have $m(b(K / F))=m(b)+(p-1)\left(b_{1}-b\right)$. 
Since $b_{1} \leqq \frac{p e}{p-1},(p-1) b-m(b) \leqq p e-m(b(K / F))$. As is easily seen, $b<b_{1}$ means $(b, p)=1$. Put $b=p\left[\frac{b}{p}\right]+p-\lambda$, so that $(p-1) b-m(b)$ $=(p-1)^{2}\left[\frac{b}{p}\right]+(p-2)(p-\lambda)$. Since $p$ is odd, then $(p-1) b-m(b)>0$, so $p e-m(b(K / F))>0$.

Next, we shall consider remaining cases. From the above equalities ii) and iii), we have $m(b(K / F)) \leqq m(b)$. Then, by the well known fact. that $m(b) \leqq e$, we have $m(b(K / F))<p e$. Thus the proof is completed.

Now, let $L / k$ be a cyclic totally ramified extension of degree $p^{n}$ with $n$ ramification numbers $b_{1}, b_{2}, \cdots, b_{n}$.

LEMma 4. Let $L / k, b_{1}$ and $b_{n}$ be as above. Then $m\left(b_{1}\right)<e$ if and only if $m\left(b_{n}\right)<p^{n-1} e$.

Proof. From [4] Lemma 2, we have that if $m\left(b_{1}\right)<e$, then $m\left(b_{n}\right)$ $<p^{n-1} e$. Then, to complete the proof, we need to show that if $m\left(b_{n}\right)$ $<p^{n-1} e, m\left(b_{1}\right)<e$. For it, as is easily seen, it suffices to prove only for the case of $n=2$. From [4] Lemma 1, we can assume that $k$ contains a primitive $p$-th root of unity without loss of generality of proof. Then we observe that $m\left(b_{1}\right)=e$ if and only if $b_{1}=\frac{p e}{p-1}$ or $\frac{p e}{p-1}-1$. Hence $b_{2} \geqq \frac{p^{2} e}{p-1}-1$. From [5] Corollary 26, we have that if $b_{1}<\frac{e}{p-1}$, $\frac{p^{2} e}{p-1}-(p-1) b_{1} \geqq b_{2}$ and if $b_{1} \geqq \frac{e}{p-1}, b_{2}=b_{1}+p e$. First, suppose $b_{1}$ $<\frac{e}{p-1}$. Then we have that $\frac{p^{2} e}{p-1}-(p-1) b_{1} \geqq \frac{p^{2} e}{p-1}-1$ and hence $(p-1) b_{1} \leqq 1$, which is a contradiction. Thus we have that $b_{1} \geqq \frac{e}{p-1}$ and $b_{1} \geqq \frac{p e}{p-1}-1$ because $b_{1}=b_{2}-p e$ and $b_{2} \geqq \frac{p^{2} e}{p-1}-1$. From this result, it clearly follows that $m\left(b_{1}\right)=e$.

4.

Throughout the rest of this paper, we assume that $k$ contains a primitive $p$-th root of unity. Then $(p-1)$ divides $e$ and so let $e_{0}$ be 
$e_{0}=\frac{e}{p-1}$. Let $\pi_{0}$ be a prime element of $k$ and denote by $\operatorname{val}_{k}$ the valuation of $k\left(\operatorname{val}_{k}\left(\pi_{0}\right)=1\right)$. Let $K / k$ be a totally ramified extension whose Galois group is a elementary abelian $p$-group of order $p^{2}$ as described in the paragraph preceding Lemma 3 . Now we may divide such extensions into following five types.

(i ) $K=k\left(w_{1}, w_{2}\right)$, where $w_{i}^{p} \in k$ and $\operatorname{val}_{k}\left(w_{i}^{p}-1\right) \geqq 2$ for $i=1,2$.

(ii) $K=k(z, w)$, where $w^{p} \in k, \operatorname{val}_{k}\left(w^{p}-1\right) \geqq 2, z^{p} \in k$ and $\operatorname{val}_{k}\left(z^{p}-1\right)$ $=1$.

(iii) $K=k\left(z_{1}, z_{2}\right)$, where $z_{i}^{p} \in k$ and $\operatorname{val}_{k}\left(z_{i}^{p}-1\right)=1$. Moreover, let $K / k$ be the extension with exactly one ramification number $p e_{0}-1$.

(iv) $K=k(\pi, w)$, where $w^{p} \in k, \operatorname{val}_{k}\left(w^{p}-1\right) \geqq 2, \pi^{p} \in k$ and $\operatorname{val}_{k}\left(\pi^{p}\right)$ $=1$.

(v) $K=k(\pi, z)$, where $z^{p} \in k, \operatorname{val}_{k}\left(z^{p}-1\right)=1, \pi^{p} \in k$ and $\operatorname{val}_{k}\left(\pi^{p}\right)$ $=1$.

In the following, we shall prove that the ring $\mathfrak{D}$ of all integers in $K$ is an indecomposable $\mathfrak{o}[G]$-module. Let $\varphi$ be an $\mathfrak{D}[G]$-endomorphism of $\mathfrak{O}$ such that $\varphi^{2}=\varphi$. Clearly, proving that the ring $\mathfrak{O}$ is indecomposable is equivalent to showing $\varphi=1$. We shall show the latter for the extension of each type stated in the above as (ii), (iii), (iv) and (v). Now we begin with the case of type (iii).

( I) The case of type (iii). Let $\Pi$ be a prime element of $K$. Since $\operatorname{val}_{K}\left(z_{i}-1\right)=p$ because of the definition of type (iii), then there exist units $\omega_{1}$ and $\omega_{2}$ of $k$ such that $z_{i}-1 \equiv \omega_{i} \Pi^{p}\left(\Pi^{p+1}\right)$ for $i=1,2$.

Lemma 5. Let $z_{i}$ and $\omega_{i}$ be as above. For rational integers $i_{1}$ and $i_{2}$, let $i_{1} \omega_{1}+i_{2} \omega_{2} \equiv 0(I I)$. Then $i_{1} \equiv i_{2} \equiv 0(p)$.

Proof. From the assumption, we have $z_{1}^{i_{1}} z_{2}^{i_{2}} \equiv 1\left(\Pi^{p+1}\right)$. Suppose $z_{1}^{i_{1}} z_{2}^{i_{2}}$ $\notin k$ and let $b$ be the ramification number for the extension $k\left(z_{1}^{i_{1}} z_{2}^{i_{2}}\right) / k$. Then, from the result of B. F. Wyman ([5]), we have $b<p e_{0}-1$, which is contrary to the fact that $K / k$ has exactly one ramification number $p e_{0}-1$. Hence $z_{1}^{i_{1}} z_{2}^{i_{2}} \in k$, which implies that $i_{1} \equiv i_{2} \equiv 0(p)$.

Lemma 6. Let $I_{0}$ and $I_{1}$ be subsets of $\{0,1, \cdots, p-1\}$. Moreover, suppose that $I_{1}$ is a proper subset. Then

$$
\operatorname{val}_{K}\left[\left\{\left(\sum_{i \in I_{0}} z_{1}^{i}\right) z_{2}\right\}-\sum_{i \in I_{0}} z_{1}^{i}\right]-\left(\left|I_{1}\right|-\left|I_{0}\right|\right)=p
$$


where $\left|I_{j}\right|$ is the number of the set $I_{j}$.

Proof. Since $z_{j}^{i} \equiv 1+i \omega_{j} \Pi^{p}\left(\Pi^{p+1}\right)$, we have

$$
\begin{aligned}
& \left\{\left(\sum_{i \in I_{1}} z_{1}^{i}\right) z_{2}-\sum_{i \in I_{0}} z_{1}^{i}\right\}-\left(\left|I_{1}\right|-\left|I_{0}\right|\right) \\
& \equiv\left|I_{1}\right| \omega_{2} \Pi^{p}+\left\{\left(\sum_{i \in I_{1}} i\right)-\left(\sum_{i \in I_{0}} i\right)\right\} \omega_{1} \Pi^{p}\left(\Pi^{p+1}\right) .
\end{aligned}
$$

By Lemma 5 and from the assumption $0<\left|I_{1}\right|<p$, it follows that $\left|I_{1}\right| \omega_{2}$ $+\left\{\left(\sum_{i \in I_{1}} i\right)-\left(\sum_{i \in I_{0}} i\right)\right\} \omega_{1} \notin\left(\pi_{0}\right)$. This completes the proof.

Now we can assume that $\varphi(1)=1$ (replacing $\varphi$ by $1-\varphi$ if necessary). Clearly $\varphi\left(z_{1}^{i_{1}} z_{2}^{i_{2}}\right)=z_{1}^{i_{1}} z_{2}^{i_{2}}$ or 0 . Let $\alpha_{j}=\frac{1}{\pi_{0}}\left(1+z_{1}+\cdots+z_{1}^{p-1}\right)\left(z_{2}^{j}-1\right)$ for $1 \leqq j<p . \quad$ Then $\alpha_{j}=\frac{1}{\pi_{0}} \cdot \frac{z_{1}^{p}-1}{z_{1}-1}\left(z_{2}^{j}-1\right) . \quad$ As $\operatorname{val}_{K}\left(z_{1}-1\right)=\operatorname{val}_{K}\left(z_{2}-1\right)$ $=p, \operatorname{val}_{K}\left(\alpha_{j}\right)=0 . \quad$ Set $\varphi\left(\alpha_{1}\right)=\frac{1}{\pi_{0}}\left\{\left(\sum_{i \in I_{1}} z_{1}^{i}\right) z_{2}-\sum_{i \in I_{0}} z_{1}^{i}\right\}$ and suppose that $I_{1}$ is a proper subset of $\{0,1, \cdots, p-1\}$. From Lemma 6 , we have $\operatorname{val}_{K}\left(\varphi\left(\alpha_{1}\right)\right)$ $\leqq p-p^{2}$, which is a contradiction. Hence $I_{1}=\varnothing$ or $\{0,1, \cdots, p-1\}$. Next suppose $I_{1}=\varnothing$, so $\varphi\left(\alpha_{1}\right)<0$, a contradiction. Thus we conclude $I_{1}=\{0,1, \cdots, p-1\}$. Now we examine the set $I_{0}$ and suppose $0<\left|I_{0}\right|<p$. Then we have $\pi_{0} \varphi\left(\alpha_{1}\right) \equiv-\left|I_{0}\right|\left(\Pi^{p}\right)$ and so $\operatorname{val}_{K}\left(\varphi\left(\alpha_{1}\right)\right)<0$, a contradiction. As $\varphi(1)=1,\left|I_{0}\right|>0$ and hence $I_{0}=\{0,1, \cdots, p-1\}$. Therefore we have $\varphi\left(z_{1}^{i} z_{2}\right)=z_{1}^{i} z_{2}$ and $\varphi\left(z_{1}^{i}\right)=z_{1}^{i}$ for $0 \leqq i<p$. Similarly, evaluating $\operatorname{val}_{K}\left(\varphi\left(\alpha_{j}\right)\right)$, we have that $\varphi\left(z_{1}^{i} z_{2}^{j}\right)=z_{1}^{i} z_{2}^{j}$ for any $i$ and any $j$, and that $\varphi=1$.

(II) The case of type (ii). Let $\alpha_{j}=\frac{1}{\pi_{0}} z^{j}\left(1+w+\cdots+w^{p-1}\right)$ for $j=0,1, \cdots, p-1$ and let $\beta=\frac{1}{\pi_{0}}\left(1+z+\cdots+z^{p-1}\right) \pi_{1}$, where $\pi_{1}$ is a prime element of $k(w)$. Using the similar arguments as in (I), we can easily conclude $\varphi=1$.

(III) The case of type (iv). Without loss of generality of proof, we can assume $\pi^{p}=\pi_{0}$. Let $\alpha_{j}=\frac{1}{\pi_{0}} \pi^{j}\left(1+w+\cdots+w^{p-1}\right)$ for $j=0,1, \cdots$, $p-1$. Using the result of S. Amano ([1]), we shall define an integer $\beta$. From his result, there exists a prime element $\pi_{1}$ of $k(w)$ such that $\pi_{1}$ is a root of the following equation

$$
X^{p}-\omega \pi_{0}^{m} X-\pi_{0}\left(1+a \pi_{0}\right)=0,
$$


where $\omega$ is a unit of $k, a \in \mathfrak{D}$ and $m=m(b(k(w) / k))$. Clearly $\pi_{1}^{p}=\pi_{0}(1$ $\left.+\omega \pi_{0}^{m-1} \pi_{1}^{2}+a \pi_{0}\right)$. Then chose an integer $\varepsilon$ of $\mathfrak{o}$ as follows: if $m \leqq 2, \varepsilon=0$ and if $m \geqq 3$, chose $\varepsilon$ such that $\pi_{1}^{p}\left(1+\varepsilon \pi_{1}\right)^{p} \equiv \pi_{0}\left(\pi_{0}^{3}\right)$. Let $u=\frac{\pi_{1}\left(1+\varepsilon \pi_{1}\right)}{\pi}$ and $\beta=\frac{\pi^{p-1}}{\pi_{0}}\left(u^{p-1}+u^{p-2}+\cdots+1\right)$. Then $u$ is a unit of $K$ such that $u \equiv 1(\Pi) . \quad$ Put $i=\operatorname{val}_{K}(u-1) . \quad$ Then it is easy to see that if $i<\frac{p^{2} e}{p-1}$ $\operatorname{val}_{K}\left(u^{p}-1\right)=p i$ and if $i \geqq \frac{p^{2} e}{p-1}, \operatorname{val}_{K}\left(u^{p}-1\right)=i+p^{2} e$. From (3), we have that if $m=1, \lambda \geqq 2$ and so $i \geqq 2$. First, assume $i<\frac{p^{2} e}{p-1}$. Then $\operatorname{val}_{K}(\beta)=p i+(p-1) p-i-p^{2}=(p-1) i-p$. As $i \geqq 2$, we have $\operatorname{val}_{K}(\beta)>0$. For the case $i \geqq \frac{p^{2} e}{p-1}, \operatorname{val}_{K}(\beta)=p^{2} e-p$. Hence $\beta$ is in $\mathscr{O}$ for the both cases. Also, we immediately get $\operatorname{val}_{K}\left(\alpha_{j}\right) \geqq 0$. Since $\varphi\left(\alpha_{j}\right)$ and $\varphi(\beta)$ are in $\mathfrak{D}$, we have that $\varphi\left(\pi^{j} w^{i}\right)=\pi^{j} w^{i}$ and $\varphi=1$ as in $(\mathrm{I})$.

(VI) The case of type (v). As $\operatorname{val}_{K}\left(z^{p}-1\right)=1, z$ satisfies the following congruence $z^{p} \equiv 1+\varepsilon_{0} \pi_{0}\left(\pi_{0}^{2}\right)$, where $\varepsilon_{0}$ is a unit of $k$. Then there exists a unit $\varepsilon$ of $k$ such that $\varepsilon^{p} \varepsilon_{0} \equiv 1\left(\pi_{0}\right)$. Now, let $\alpha_{0}=\frac{1}{\pi_{0}}\left[\{\varepsilon(-1+z)\}^{p-1}\right.$ $\left.+\{\varepsilon(-1+z)\}^{p-2} \pi+\cdots+\pi^{p-1}\right]$ and $u=\frac{\varepsilon(-1+z)}{\pi}$. Then $u^{p} \equiv \frac{\varepsilon^{p}\left(-1+z^{p}\right)}{\pi_{0}}$ $\equiv 1\left(\Pi^{p^{2}}\right)$. Put $i=\operatorname{val}_{K}(u-1)$, so clearly $i \geqq p$. Then we have $\operatorname{val}_{K}\left(\alpha_{0}\right)$ $>0$ as in (III). We observe easily that $(-1+z)^{p-1} \equiv 1+z+\cdots+z^{p-1}(p)$. Hence $\pi_{0} \varphi\left(\alpha_{0}\right) \equiv \varepsilon^{p-1} \varphi\left(1+z+\cdots+z^{p-1}\right)(\pi)$. Set $\varphi\left(1+z+\cdots+z^{p-1}\right)$ $=\sum_{i \in I} z^{i}$ and suppose $1 \leqq|I|<p$. Then $\varphi\left(1+z+\cdots+z^{p-1}\right)$ is a unit of $K$ and so $\operatorname{val}_{K} \varphi\left(\alpha_{0}\right)=-p^{2}$, a contradiction. Thus $\varphi\left(z^{i}\right)=z^{i}$ for $i=0$, $1, \cdots, p-1$. Using the same arguments as in (III) with this fact, we conclude $\varphi=1$.

Proposition 1. Suppose that $k$ contains a primitive p-th root of unity. Let $K / k$ be a totally ramified extension whose Galois group is an elementary abelian $p$-group of order $p^{2}$. Then $\mathfrak{D}$ is an indecomposable $\mathfrak{o}[G]$-module.

Proof. We have just proved the results for the cases where the extensions $K / k$ are not of type (i). It remains to verify for the case of type (i). First, we note that for any subfield $F$ of degree $p$ in $K, m(b(F / k))<e$. From [4] Theorem 3, the ring $\mathfrak{D}_{F}$ of all integers in $F$ is indecomposable. 
Hence, from Lemma 1, we obtain the desired result for the extension of type (i).

4.

In this section, we shall treat the case where the Galois group $G$ is a direct product of two cyclic groups whose orders are $p$ and $p^{n}$ respectively. Let $F$ and $L$ be cyclic totally ramified extensions of degrees $p$ and $p^{n}$ respectively. Let $K$ be a composition field of $F$ and $L$, and assume that $K$ is totally ramified. Let $\varphi$ be an $\mathfrak{o}[G]$-endomorphism of $\mathscr{O}$ such that $\varphi^{2}=\varphi$ as in the previous section. As $G$ is abelian, we can consider $\varphi$ as an idempotent of $k[G]$. Let $L_{1}$ is the unique subfield of degree $p$ in $L$ and $S$ denote the subgroup of $G$ corresponding to $L_{1}$. First, we assume that $k$ contains a primitive $p^{n}$-th root of unity. Then there exists an element $\gamma$ of $L$ such that $L=k(\gamma)$ and $\gamma^{p^{n}}=\pi_{0}^{p^{m}} u_{0}$, where $0 \leqq m$ $\leqq n$ and $u_{0}$ is a unit of $k$ such that $u_{0} \equiv 1\left(\pi_{0}\right)$. Denote by $\delta$ a primitive element of $F$ as given in $\S 3$, i.e. $\delta$ is one of $w, z$ and $\pi$. Now, since $k \gamma^{i} \delta^{j}$ is a $k[G]$-module, obviously $\varphi\left(\gamma^{i} \delta^{j}\right)=\gamma^{i} \delta^{j}$ or 0 for $0 \leqq i<p^{n}$ and $0 \leqq j<p$. For $1 \leqq i<p^{n-1}$ with $(i, p)=1$, put $q=\left[i p^{m} / p^{n}\right]$. Then $\gamma^{i} / \pi_{0}^{q}$ is integer of $K$. For the case $m \geqq 1$, let $v=\gamma^{p^{n-1}} / \pi_{0}^{p^{m-1}}$. Immediately, we have that $v^{p}$ is a unit of $k$ such that $v^{p} \equiv 1\left(\pi_{0}\right)$ and that $\sum_{l=0}^{p-1} \mathfrak{o}\left(\frac{\gamma^{i+l p^{n-1}}}{\pi_{0}^{q+l p^{m-1}}}\right)=\frac{\gamma^{i}}{\pi_{0}^{q}} \sum_{l=0}^{p-1} \mathfrak{o} v^{l}$. For the case $m=0$, we have $\sum_{l=0}^{p-1} \mathrm{D} \gamma^{i+l p^{n-1}}=\gamma^{i}\left(\sum \mathrm{o} \eta^{l}\right)$, where $\eta=\gamma^{p^{n-1}}\left(\operatorname{val}_{L_{1}}(\eta)\right.$ $=1$ ). Furthermore, we remark $\gamma^{i} L_{1}=\sum_{l=0}^{p-1} k \gamma^{i+l p^{n-1}}$. Now, from the similar arguments as in $\S 3$ with the above remarks, we conclude that $\varphi\left(\gamma^{i+l p^{n-1}} \delta^{j}\right)$ $=\gamma^{i+l p^{n-1}} \delta^{j}$ for any $l$ and any $j$, or all $\varphi\left(\gamma^{i+l p^{n-1}} \delta^{j}\right)$ are simultaneously equal to the zero element of $\mathcal{D}$ except the case where $p=3, e=2$, $\operatorname{val}_{L}\left(\gamma^{i} / \pi_{0}^{q}\right) \geqq p^{n-1}$ and the extension $L_{1} F$ is of type (v) such that $m\left(b\left(L_{1} / k\right)\right)$ $=2$ and $m(b(F / k))=3$. In the following, we consider the remaining case. Let $\pi_{1}$ be a prime element of $L_{1}$ which satisfies the equation (4) as given in $\S 3$. As is easily seen, $\left\{1, v, v^{2}\right\}$ is an integral base. Then $\pi_{1}$ is written in the form $\pi_{1}=a_{0}+a_{1} v+a_{2} v^{2}$, where $a_{i} \in \mathfrak{D}$. As $\operatorname{tr}_{L_{1} / k} \pi_{1}=0$, we see that $a_{0}=0$ and $a_{1} \equiv-a_{2} \neq 0\left(\pi_{0}\right)$. Denote $\gamma^{i} / \pi_{0}^{q}$ by $\gamma^{\prime}$ and let $i_{0}$ be the minimal integer $i^{\prime}$ such that $3^{n-1} i^{\prime}+\operatorname{val}_{L}\left(\gamma^{\prime}\right) \geqq 3^{n}$. As $3^{n-1} \leqq \operatorname{val}_{L} \gamma^{\prime}<3^{n}$, we have $0<i_{0} \leqq 2$. First, we consider the case $i_{0}=1$. Let $\alpha_{0}=\frac{\gamma^{\prime}}{\pi_{0}}\left(1+v+v^{2}\right)$ and $\alpha_{2}=\frac{\gamma^{\prime} \pi^{2}}{\pi_{0}^{2}}\left(1+v+v^{2}\right)$. Then, evaluating $\operatorname{val}_{K}\left(\varphi\left(\alpha_{0}\right)\right)$ and $\operatorname{val}_{K}\left(\varphi\left(\alpha_{2}\right)\right)$, 
we have $\varphi\left(\gamma^{\prime} v^{i}\right)=\gamma^{\prime} v^{i}$ and $\varphi\left(\gamma^{\prime} v^{i} \pi^{2}\right)=\gamma^{\prime} v^{i} \pi^{2}$ for $0 \leqq i<3$. Next let $\alpha_{1}$ $=\frac{\gamma}{\pi_{0}}\left(1+u+u^{2}\right)$, where $u=\frac{\pi_{1}}{\pi}$ as in $\S 3$. As $u \equiv 1\left(\pi_{1}\right)$, we see $\operatorname{val}_{K} \alpha_{1} \geqq 0$. Clearly $\frac{\pi_{0}}{\pi_{0}} \alpha_{1}=\frac{\gamma}{\pi_{0}^{2}}\left(\pi_{0}+\pi_{1} \pi^{2}+\pi_{1}^{2} \pi\right)$. Let $\varphi\left\{\gamma\left(1+v+v^{2}\right) \pi\right\}=\gamma \pi\left(\sum_{i \in I} v^{i}\right)$ and suppose $1 \leqq|I|<3$. Then $\pi_{0}^{2} \varphi\left(\alpha_{1}\right) \equiv \gamma\left(-a_{1}^{2}\right) \pi\left(\gamma \pi_{0}\right)$, which is contrary to $\varphi\left(\alpha_{1}\right) \geqq 0$ since $a_{1}$ is a unit of $k$ and $\operatorname{val}_{K}(\gamma \pi)<2 \cdot 3^{n+1}$. Thus we conclude $I=\{0,1,2\}$ or $\varnothing$. Similarly, for the case $i_{0}=2$, we have the desired result. This completes the proof of the above statement for this case. Now, according to the same arguments as used in [4] with the above statement, we have that the idempotent $\varphi$ is an element of $k[S]$. Next, we assume that $k$ does not contains a primitive $p^{n}$-th root of unity. Then it follows from [4] Lemma 4 that $\varphi \in k[S]$. Therefore, clearly by the inductive arguments, we obtain the following proposition.

Proposition 2. Let $K / k$ be a totally ramified extension whose Galois group is a direct product of two cyclic groups of orders $p$ and $p^{n}$ respectively. Suppose that $k$ contains a primitive $p$-th root of unity. Then $\mathfrak{D}$ is indecomposable.

5.

In this section, we shall give the proofs of Theorem 1 and Theorem 2. First, we shall prove Theorem 1 and use the same notations as in the previous sections. Let $G$ be a non-cyclic $p$-group of order $p^{n}$ and let $E$ be a central idempotent of $k[G]$ such that $E \subseteq \subseteq \mathfrak{D}$. We use induction on $n$ of $p$-power $p^{n}$. From Proposition 1, we obtain the result for $n=2$. Assume the result holds for $n<r$. Let $G$ be a non-cyclic $p$-group of order $p^{r}$. First, we assume that the center $C(G)$ of $G$ is not cyclic as in Lemma 1. Now, if there exists a subgroup $H$ of order $p$ in $C$ such that the factor group $G / H$ is cyclic, then $G$ is of type $\left(p, p^{r-1}\right)$ and so the desired result follows from Proposition 2. Thus we consider the case where for any subgroup $H$ of order $p$ in $C$, the factor group $G / H$ is not cyclic. By our inductive assumption, $f_{H}(E)=1$. Then, from Lemma 1 , we obtain $E=1$, which completes the proof of Theorem 1 for the first case. Next, we assume that $C(G)$ is cyclic. As $G$ is not cyclic, $G$ is not abelian and hence $G / Z$ is not cyclic. By our inductive assumption, we have $f_{z}(E)=1$ and so we can write $E=\frac{1}{p} \sum_{i=0}^{p-1} z^{i}+E_{0}$, where $E_{0}$ is a 
central idempotent such that $E_{0} \cdot \frac{1}{p}\left(\sum_{i=0}^{p-1} z^{i}\right)=0$. From Lemma 2, we have $E_{0} \in C_{G}(b) \Phi$ and $E \in C_{G}(b) \Phi$. Denote by $K_{Z}$ the subfield corresponding to $Z$. Then, from Lemma $3, m\left(b\left(K / K_{Z}\right)\right)<e_{z}$, where $e_{Z}$ is the absolute ramification index of $K_{Z}$, since $K_{Z}$ contains a subfield $K_{B}$ corresponding to $B$. Therefore, if $C_{G}(b) \Phi$ is cyclic, it follows from Lemma 4 and [4] Theorem 3 that $E=1$. Thus we now consider the case where $C_{G}(b) \Phi$ is not cyclic. Now, by (2), we note $\left|C_{G}(b) \Phi\right|<|G|$. Hence we can apply our inductive assumption to this case and conclude $E=1$. The proof of Theorem 1 is completed.

Next, we shall prove Theorem 2. Let $\varphi$ be an $\mathrm{o}[G]$-endomorphism of $\mathfrak{S}$ such that $\varphi^{2}=\varphi$. To prove Theorem 2 it is sufficient to show $\varphi=1$. As the extension $K / k$ is abelian, we can consider $\varphi$ as an idempotent of $k[G]$. Then, from Theorem 1, we have obtained $\varphi=1$ and the proof of Theorem 2.

\section{REFERENCES}

[1] S. Amano, Eisenstein equations of degree $p$ in a p-adic field, J. Fac. Sci. Univ. Tokyo 18, No. 1 (1971), 1-21.

[2] B. Huppert, Endlich Gruppen I, Die Grundlehren der math. Wissenshaften, Band 134, Springer-Verlag, Berlin and New York 1967.

[ 3 ] E. Maus, Arithmetish disjucte Körper, J. reine angew. Math. 226 (1967), 184203.

[ 4 ] Y. Miyata, On the module of a cyclic extension over a p-adic number field, Nagoya Math. J. 73 (1979), 61-68.

[ 5 ] B. F. Wyman, Wildly ramified gamma extension, Amer. J. of Math. 91 (1969), $135-152$. 